Abstract FRI0428 - Table 2. Associations between mean number of capillaries at baseline and disease activity (ESSG score 2003) at FU (linear regression)

\begin{tabular}{lcc}
\hline Predictors & B $(95 \%$ Cl) & p \\
\hline m_nr/pat rater 2 & $-0.45(-0.834,--$ & 0.02 \\
m_nr/3rd dom rater 2 & $0.07)$ & \\
m_nr/4th non-dom rater & $-0.33(-0.62,-0.03)$ & 0.03 \\
2 & $-0.27(-0.57,0.02)$ & 0.07 \\
\hline
\end{tabular}

Conclusions: The mean number of capillaries had a good association with the history of DUs and predicted disease activity at 6 months follow-up. The $\mathrm{m}$ nr/pat performed better in our analysis than the $\mathrm{m} \_n r / 3 r d$ dom and $m \_n r / 4$ th non-dom, however these could be used alternatively in clinical practice as they are less time consuming.

Acknowledgements : This work is part of the QUANTICAP project

Disclosure of Interest: None declared

DOI: 10.1136/annrheumdis-2018-eular.7054

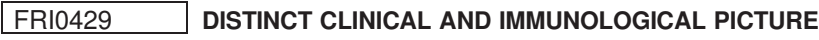 OF MCTD PATIENTS WITH SKIN INVOLVEMENT}

A. Felis-Giemza ${ }^{1}$,E. Kontny ${ }^{2}$, J. Nałęcz-Janik ${ }^{1}$, K. Walkiewicz-Pielaszek ${ }^{1}$ Z. Czuszyńska ${ }^{3}$, Z. Zdrojewski ${ }^{3}$, A. Paradowska-Gorycka ${ }^{4}$, M. Olesińska ${ }^{1}$. ${ }^{1}$ Connective Tissue Disease Department, ${ }^{2}$ Department of Patophysiology and Immunology, National Institute of Geriatrics, Rheumatology and Rehabilitation, Warsaw Poland, Warsaw; ${ }^{3}$ Clinic of Internal Medicine, Connective tissue Disease andGeriatrics, Medical University of Gdansk, Gdańsk; ${ }^{4}$ Department of Biochemistry and Molecular Biology, National Institute of Geriatrics, Rheumatology and

Rehabilitation, Warsaw Poland, Warsaw, Poland

Background: Mixed connective tissue disease (MCTD) is characterised by the co-existence of systemic lupus erythematosus (SLE), rheumatoid arthritis (RA), systemic sclerosis (SSc) and polymyositis/dermatomyositis (PM/DM) symptoms. The majority of patients have skin symptoms typical of at least one of the diseases making up the clinical picture MCTD. MCTD is characterised by an auto-reactive antibody response to RNP antigen and resultant formation of anti-U1RNP antibodies. Current knowledge on cytokine biology and their documented role in the pathogenesis of SLE, SSc and PM/DM suggests that also in MCTD some of them may affect clinical course, activity and/or degree of organ damage.

Objectives: To compare clinical and immunological characteristics of MCTD patients with/without skin involvement.

To identify clinical and immunological parameters increasing the risk for a specific (SLE- or SS-like) type of skin lesions and protecting against them.

Methods: 79 MCTD patients based Kasukawa' MCTD diagnostic criteria were included. The patients were divided into groups based on the presence of skin lesions typical for a given MTCD component: SLE- SSc- and DM-specific.

Results: Skin lesions were found in the majority of the MCTD patients (81\%). The SLE-, SSc- and DM-specific skin symptoms were found in $54 \%, 61 \%$ and $5 \%$ of the patients, respectively. The group of patients with skin symptoms typical of DM was to small to distinguish it separately (4/79). The measures of disease activity (mean $\mathrm{Al}=10.6$ vs $5.5 ; \mathrm{p}=0.006$ ) and MCTD-related damage ( $\mathrm{Dl}=4.1$ vs 2.1 ; $\mathrm{p}=0.009$ ) in patients with skin involvement were twice as high as in individuals with the intact skin. Furthermore, patients with skin involvement had higher mean serum concentrations of TNF- $\alpha$ (46.4 vs $2.3 \mathrm{pg} / \mathrm{ml} ; \mathrm{p}=0.013)$, and lower levels of IFN- $\gamma$ (43.2 vs $1208 \mathrm{pg} / \mathrm{ml} ; \mathrm{p}<0.001)$ than patients without any skin symptoms.

The following clinical and immunological parameters were shown to be independently associated with specific types of skin involvement in MCTD patients on multivariate logistic regression analysis:

Independent risk factors for:

- SLE-like skin changes: increased ESR ( $O R=8.9,4.47$ and 2.6, respectively), higher Al scores and swelling of the hands,

- SSc-like skin changes: higher DI scores $(\mathrm{OR}=1.522)$, and presence of anti-Ro60 antibodies (OR=15.903)

Independent protective factors for:

- SLE-like MCTD: chronic progressive course of the disease $(O R=0.248)$ and higher serum concentration of IFN- $\gamma(\mathrm{OR}=0.988)$

- SSc-like MCTD: acute onset of the disease (OR=0.155).

Conclusions: The course of MCTD in patients with skin involvement is more severe with specific panel of cytokine levels (increased TNF- $\alpha$ and decreased IFN- $\gamma$ serum concentrations) is characteristic, as compared to patients with the intact skin.

In patients with SLE-like skin lesions MCTD is more often multiphasic, its clinical activity and levels of inflammatory markers are higher while serum concentration of IFN- $\gamma$ is diminished. Patients with SSc-like skin lesions more often have chronic MCTD, associated with more severe organ damage and elevated serum levels of TNF- $\alpha$.
Disclosure of Interest: None declared

DOI: 10.1136/annrheumdis-2018-eular.7190

\section{FRI0430 EFFECT OF ORAL NUTRITIONAL INTERVENTION ON NUTRITIONAL STATUS IN PATIENTS WITH SYSTEMIC SCLEROSIS}

A. Wojteczek ${ }^{1}$, M. Ziętkiewicz ${ }^{1}$, S. Małgorzewicz ${ }^{2}$, Z. Zdrojewski ${ }^{1}{ }^{1}$ Department of Internal Medicine, Connective Tissue Diseases and Geriatrics; ${ }^{2}$ Department of Clinical Nutrition, Medical University Of GdanSk, Gdańsk, Poland

Background: Patients with systemic sclerosis (SSc) are at risk of malnutrition which ranges from $18 \%$ to $56 \%$ of cases. ${ }^{1,2}$ The high impact of nutritional status on clinical outcome has been shown for many diseases. The optimal nutritional treatment can lead to improvement or preservation of the current nutritional status and increases probability of long-term survival. ${ }^{3}$

Objectives: The aim of the study was to determine whether nutritional support has an impact on improvement of nutritional status in SSc patients.

Methods: The study included 61 patients with SSc and 49 healthy adults. Nutritional status was determined with subjective global assessment (SGA), body mass index (BMI), bioelectrical impedance analysis (BIA) and anthropometric measurements. Nutrition-related laboratory tests were measured. Appetite was assessed by simplified nutritional appetite questionnaire (SNAQ).

Results: Impaired nutritional status was confirmed in 16 patients with SSc $(26,2 \%)$. Those patients had significantly lower SGA, BMI $(p=0,0019)$, hand grip strength $(p=0,0019)$, appetite $(p=0,019)$ and BIA parameters such as lean tissue mass $(p=0,013)$, intracellular water $(p=0,0006)$, adipose tissue mass $(p=0,04)$. In laboratory tests levels of haemoglobin, albumin and HDL cholesterol were significantly lower, while erythrocyte sedimentation rate (ESR) was higher $(p=0,0025)$. Thirteen patients had dietary intervention (high-energy, high-protein, oral, liquid nutritional supplements) for 12 weeks. SGA $(p=0,017)$ and hand grip strength $(p=0,006)$ improved after nutritional treatment. BMI, appetite, BIA parameters, lipid profile and ESR after 12 weeks remained stable.

Conclusions: Assessment of nutritional status in SSc patients should be performed regularly, because inclusion of oral nutritional intervention may improve SGA and hand grip strength.

\section{REFERENCES:}

[1] Baron $\mathrm{M}$, et al. Malnutrition is common in systemic sclerosis: results from the Canadian scleroderma research group database. J Rheumatol 2009;36:2737-43.

[2] Krause L, et al. Nutritional status as marker for disease activity and severity predicting mortality in patients with systemic sclerosis. Ann Rheum Dis 2010;69:1951-1957.

[3] Harrison E, et al. Long-term outcome of patients with systemic sclerosis requiring home parenteral nutrition. Clinical Nutrition 2015;34:991-996.

Disclosure of Interest: None declared

DOI: 10.1136/annrheumdis-2018-eular.6703

\section{FRI0431 DYSREGULATION OF LYMPHANGIOGENETIC FACTORS IN SYSTEMIC SCLEROSIS PATIENTS WITH PULMONARY ARTERIAL HYPERTENSION}

A.-M. Hoffmann-Vold ${ }^{1}$, H. Didriksen ${ }^{1}$, H. Fretheim ${ }^{1}$, E. Gude ${ }^{1}$,V. Palchevskiy ${ }^{2}$,

T. Garen ${ }^{1}, \varnothing$. Midtvedt ${ }^{1}$, A. Andreassen ${ }^{1}$, J. Belperio $^{2}, \varnothing$. Molberg ${ }^{1}{ }^{1}$ Oslo

University Hospital, Oslo, Norway; ${ }^{2}$ Ucla, La, USA

Background: Pulmonary arterial hypertension (PAH) continues to be a major complication in systemic sclerosis (SSc); indicating an unmet need for rationa therapeutic targets. Recently, we found that chemokine CCL21 was upregulated in SSc and associated with PAH. CCL21 appears to be a key regulator of the expression and secretion of vascular endothelial growth factor-C (VEGF-C); a critical growth factor for lymphatic vessels. This is interesting as SSc is marked by lymphatic vessel abnormalities; and high blood levels of Angiopoietin-2, which is a known regulator of VEGF-C. Moreover, it was recently shown that VEGFR3, the cognate receptor for VEGF-C, is down-regulated in pulmonary arterial endothelial cells from human idiopathic PAH subjects with BMPR2 gene defects. Based on these observations, we reasoned that the observed upregulation of CCL21 in SSc-PAH could be linked to VEGF-C and Ang-2 dysregulation.

Objectives: Assess serum concentrations of CCL21, the VEGF family and Ang-2 in right heart catheterization ( $\mathrm{RHC}$ ) verified SSc-PAH patients and compare these to patients with borderline $\mathrm{PAH}$, no $\mathrm{PH}$ and to healthy controls.

Methods: Sera from the prospective Oslo University Hospital SSc cohort $(n=372$ and healthy controls $(n=100)$ were analysed for VEGF-A,C,D, CCL21 and Ang2 using Luminex kits from Millipore. Patients with an incident $\mathrm{RHC}(\mathrm{n}=167)$ were included in the present study. $\mathrm{PAH}$ was defined as precapillary $\mathrm{PH}$ (mean 